\title{
Can A Dedicated Multidisciplinary Tumor Board Improve Personalized Medicine for Patients in Interventional Oncology? A Large Retrospective Single-Center Experience
}

\author{
(1) Roberto IEZZI, ${ }^{1}$ (1) Bruno FIONDA, ${ }^{2}$ (1) Patrizia CORNACCHIONE, 2 (1) Martina IEZZI, ${ }^{3}$

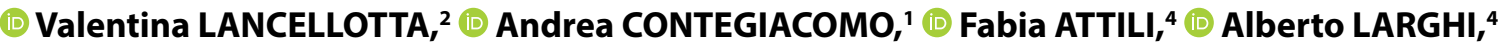 \\ (1) Francesco CELLINI, ${ }^{2}$ (1) Fabio MARAZZI, ${ }^{2}$ (D) Giuseppe COLLOCA, ${ }^{2}$ (1) Ángeles ROVIROSA, ${ }^{5}$ \\ (1) György KOVÁCS, ${ }^{6}$ (D) Maria Antonietta GAMBACORTA, ${ }^{2}$ (1) Riccardo MANFREDI, ${ }^{1}$ \\ (1) Vincenzo VALENTINI, ${ }^{2}$ (i) Luca TAGLIAFERRI ${ }^{2}$
}

\begin{abstract}
'Department of Diagnostic Imaging, Fondazione Policlinico Universitario "A. Gemelli" IRCCS, Radiation Oncology and Hematology, U.O.C. of Diagnostic and General Interventional Radiology, Rome-Italy

'Department of Diagnostic Imaging, Fondazione Policlinico Universitario "A. Gemelli" IRCCS, Radiation Oncology and Hematology, U.O.C. of Radiation Oncology Therapy, Rome-Italy

${ }^{3}$ Sacred Heart Catholic University, Institute of Radiology, Rome-Italy

${ }^{4}$ Fondazione Policlinico Universitario "A. Gemelli" IRCCS, Digestive Endoscopy Unit, Rome-Italy

${ }^{5}$ Department of Radiation Oncology, Hospital Clinic I Universitari, Barcelona-Spain

${ }^{6}$ Sacred Heart Catholic University, Educational Program Director Gemelli-INTERACTS, Rome-Italy
\end{abstract}

\section{OBJECTIVE}

Interventional oncology (IO) offers minimal invasive oncological procedures to personalize therapeutic approaches. We aimed at assessing potential advantages of an IO multidisciplinary tumor board (INTER-BOARD) in terms of waiting time, procedural adverse events, and length of hospital stay.

\section{METHODS}

We retrospectively evaluated all patients managed at our institutional INTER-BOARD from January 2018 to June 2019. Outcome measurements such as number of patients managed in each semester, median interval time between initial assessment and admission to the hospital, total number of procedures, procedures type, adverse events, and complication-related days of hospital stay were analyzed.

\section{RESULTS}

The INTER-BOARD discussed 438 patients (mean age 64 years; range 27-92) during the study period. Overall, 203 procedures were performed in patients $>65$ years and 49 in patients $>80$ years. The number of patients discussed progressively increased over time 82 (18.7\%) during the first semester, 120 (27.4\%) during the second semester, and 236 (54.1\%) in the third semester. Patients with 33 different cancer types were discussed and 22 different procedure types were performed. Mean hospitalization time was 4 days (progressively decreasing over time from 5 to 3 days). Adverse events occurred in $4 \%$ of cases, with a progressively decreasing rate over time $\left(16 \%, 5 \%\right.$, and $3 \%$ for $1^{\text {st }}, 2^{\text {nd }}$, and $3^{\text {rd }}$ semester, respectively). 


\section{CONCLUSION}

In our experience, the dedicated INTER-BOARD allowed optimization of patient care through critical evaluation of all treatment options offered by different specialists. This approach reached optimal treatment results, with low adverse events, and reduced waiting, as well as hospitalization time.

Keywords: Brachytherapy; interventional oncology; interventional radiology; multidisciplinary team; personalized medicine; radiotherapy.

Copyright $\odot$ 2022, Turkish Society for Radiation Oncology

\section{Introduction}

Oncology should offer personalized management, defined as "tailor made" treatment, which should be based on multidisciplinary assessment[1] focusing on efficacy, feasibility, and cost/benefit but, at the same time, considering the patient's age, clinical condition, type of disease and personal needs. Nowadays, minimally invasive procedures are increasingly used to manage oncological patients; these procedures are especially beneficial in elderly frail patients[2] to avoid overtreatment or age-related undertreatment.[3] Different non-surgical procedure types exist to treat localized malignancies or oligometastatic diseases, which can be collected under the name interventional oncology (IO) and include interventional radiotherapy (IRT) (brachytherapy), interventional radiology, interventional endoscopy, and interventional chemotherapy. IO is a new tool in cancer treatment and has the potential to improve treatment results and benefits, as well as to reduce complications in localized solid cancers or selected oligometastatic diseases. Many IO procedures could be proposed alternatively and only a detailed case assessment and multidisciplinary evaluation can result in the best personalized therapeutic choice.

An IO center should offer an opportunity in cancer care and specifically, "interdisciplinary service for diagnosis and treatment of cancer and cancer-related problems using targeted (focal) minimally invasive procedures."[4] Specialized multidisciplinary tumor boards (MDTs), in this paper called "IO MDT board (INTER-BOARD)," are interdisciplinary meetings in which different specialists are directly involved in patient management, overcoming the limits of a general tumor board. This evaluation aims to discover and report the influence of the INTER-BOARD in terms of waiting time, medical procedure complications, and average hospital stay.

\section{Materials and Methods}

As a consequence of a health care plan at Gemelli University Hospital a dedicated pathway for IO patients was defined (Fig. 1). From January 2018 all patients, candidates to interventional procedures, were discussed in the dedicated weekly multidisciplinary meetings (INTER-BOARD). INTER-BOARD members are radiation oncologists experienced in IRT, radiation oncologists experienced in external beam focal radiotherapy, interventional radiologists, interventional endoscopists, geriatric-oncologists, and radiation technicians. All patients were evaluated following a multidisciplinary pre-hospitalization discussion to intercept any condition that can influence the procedure and to sign the informed consent. A retrospective analysis was carried out using medical records of patients managed at the INTER-BOARD from January

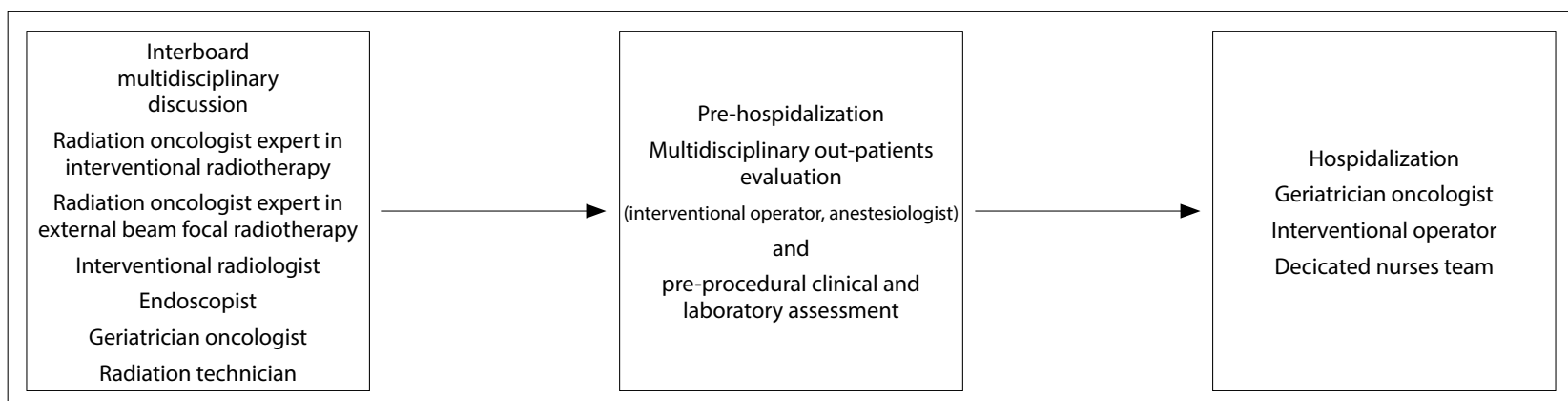

Fig. 1. A dedicated pathway for interventional oncology patients. 


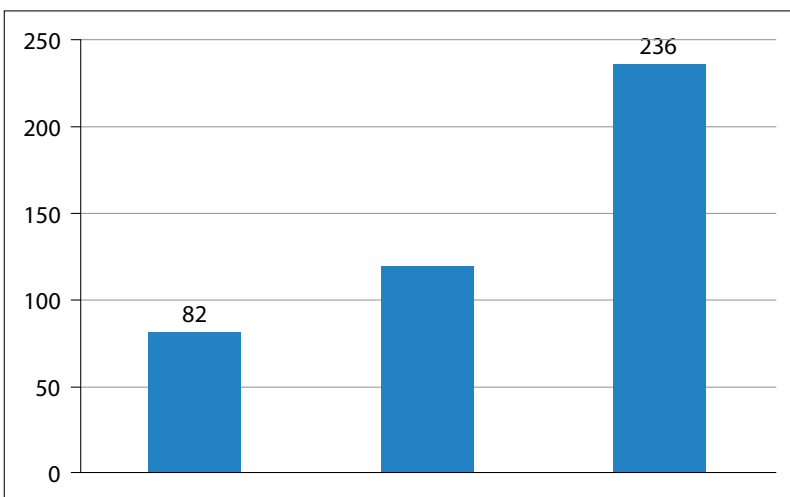

Fig. 2. Number of patients discussed during INTERBOARD: $1^{\text {st }}, 2^{\text {nd }}$ semester 2018 and $1^{\text {st }}$ semester 2019.

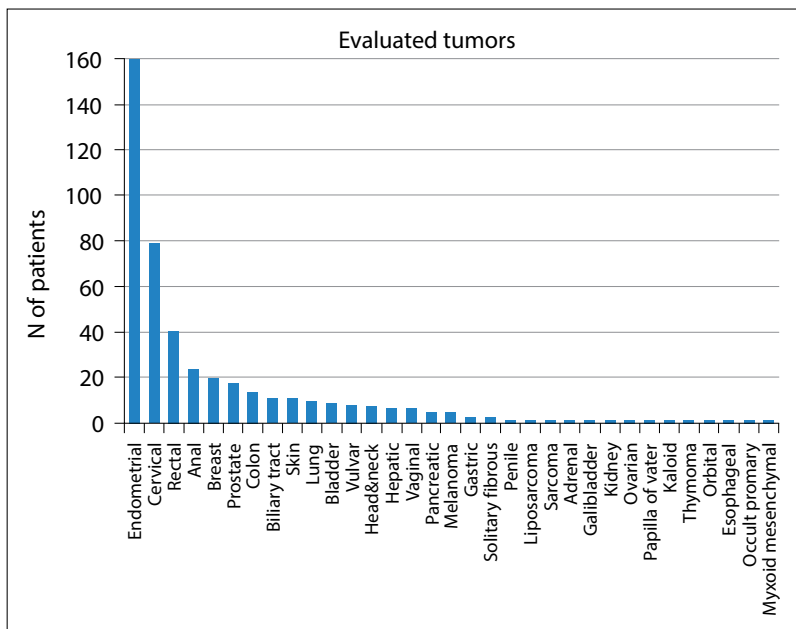

Fig. 3. Types of evaluated tumors.

2018 to June 2019. The number of patients in each semester, the median time between initial assessment and admission to the hospital, the total number of procedures, features, procedure types, side effects, length of hospital stay, and complication rates were collected and analyzed.

\section{Analysis of Data and Statistical Methods}

Clinical data were progressively collected in a database obtained by "SPEED" technology (connected with COBRA/BOA[5] informatics architecture), which is an electronic institutional platform, that is an evolution of the "SPIDER'S NET" system.[6] Data processing was carried out by two researcher (MI and PC). Patient characteristics were represented as frequencies and percentages. The statistical analysis was performed using Microsoft Excel for macOS software.

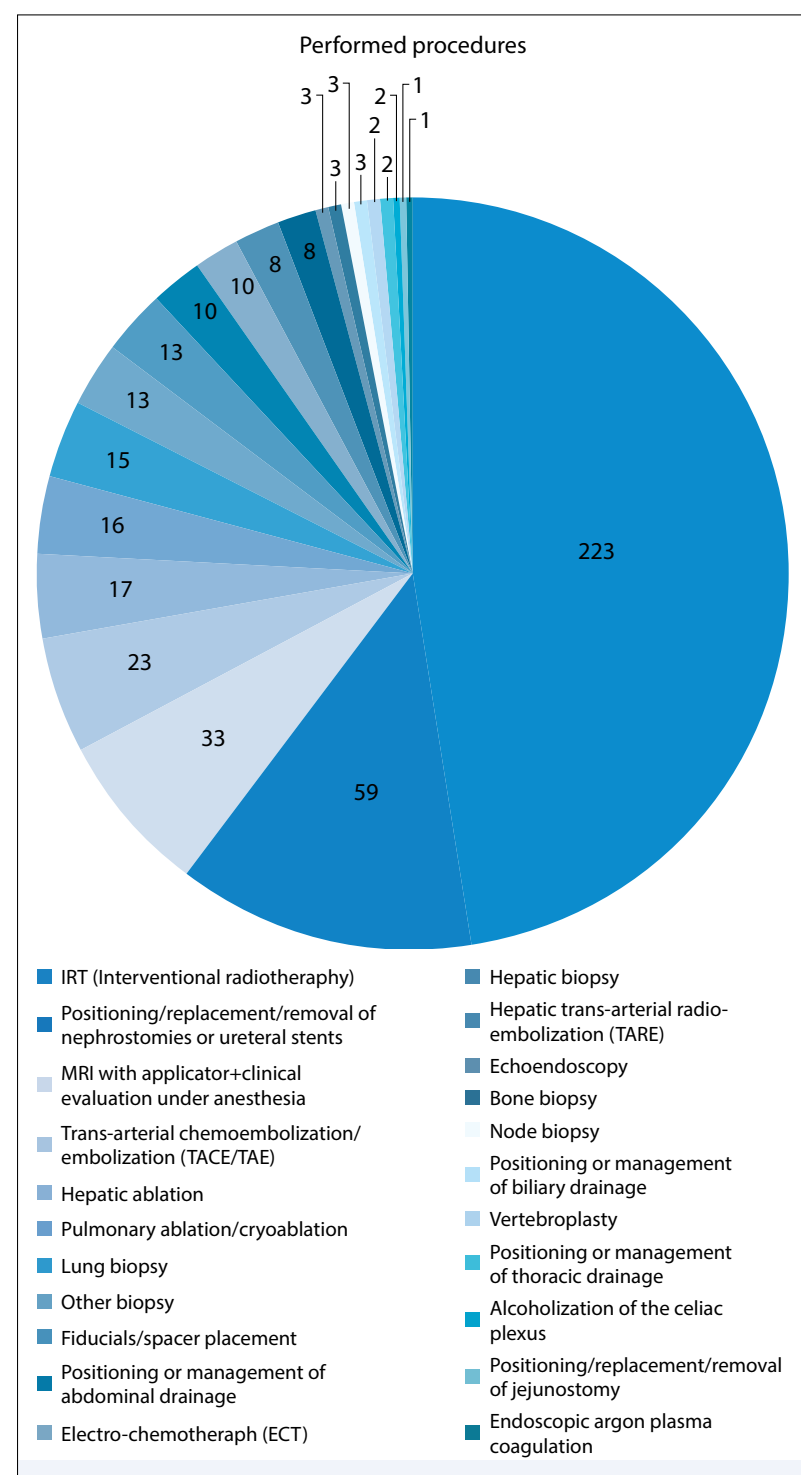

Fig. 4. Types of performed procedures (The list of procedures is reported in descending order).

\section{Results}

From January 01, 2018 to June 30, 2019, the INTER BOARD discussed 438 consecutive patients. The mean age was 64 years (range 27-92 years), with 203 procedures performed in patients over 65 years and 49 over 80 years. The number of discussed patients was progressively increased: $82(18.7 \%)$ from January to June 2018, 120 (27.4\%) from July to December 2018, and 236 (54.1\%) from January to June 2019 (Fig. 2).

Patients with 33 cancer types were discussed (Fig. 3 ) and 22 different types of procedures were performed (Fig. 4). The average waiting time from INTERBOARD to pre-hospital admission assessment was 11 


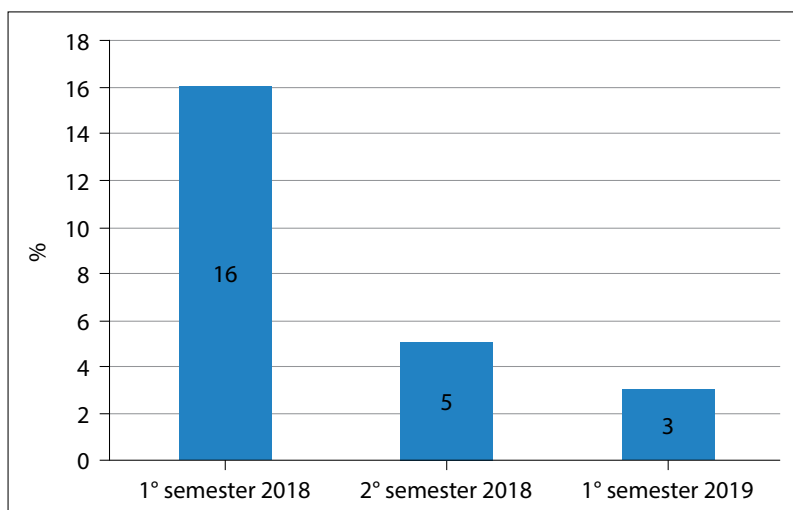

Fig. 5. Percentage of hospitalizations with a complication: $1^{\text {st }}, 2^{\text {nd }}$ semester 2018 and $1^{\text {st }}$ semester 2019.

days, while the average interval from pre-hospital assessment and admission was 11 days. The average waiting time from INTER-BOARD to hospital admission was 22 days. Urgent cases were managed in a fast-track pathway guaranteeing procedures in optimal clinical time. In selected urgent clinical cases the multidisciplinary evaluation was performed ad hoc with a complete laboratory and radiological assessment (when required) directly performed at the ward. The overall mean hospitalization time was 4 days. An analysis of hospitalization time for semesters was performed. With the improvement of the pathway and the pre-hospitalization assessment, the mean hospitalization time decreased progressively over time going from 5 days in the first semester to 4 days in the second semester and 3 days in the last semester considered.

The overall complication rate was $4 \%$. The rate of complications progressively decreased $(16 \%, 5 \%, 3 \%$, respectively, for $1^{\text {st }}, 2^{\text {nd }}$ semester 2018 and $1^{\text {st }}$ semester 2019) (Fig. 5). Figure 6 summarizes all observed complications.

Currently, the health costs related to increasingly complex patients make the sustainability of health systems difficult. In this scenario, a multidisciplinary and multidimensional approach in the IO tumor board has as its main advantage the treatments personalization. A "tailor-made" treatment, the constant multidisciplinary case management and the presence in the evaluations of an oncogeriatric assessment of the complexity, allows more targeted and punctual interventions which in clinical terms corresponds to a reduction of the phenomena of under and overtreatment, reduction of the patient's hospital stay with reduction of the indirect effects (risk of delirium, enticement, loss of performance, and infections), improvement of patient compliance and impact

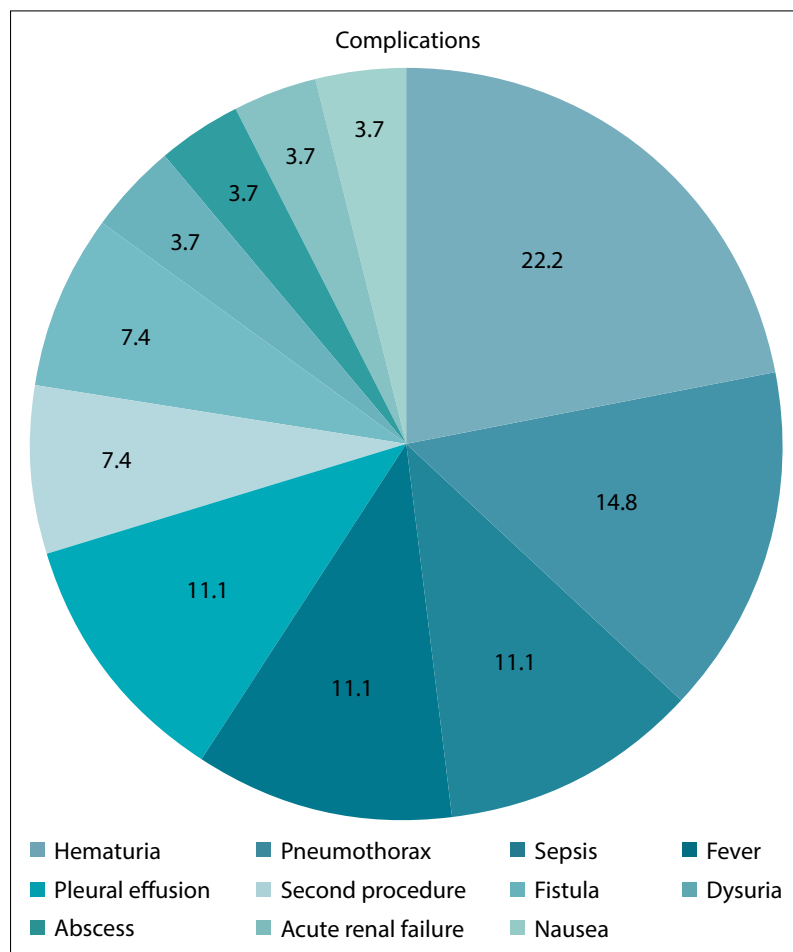

Fig. 6. Complications observed in treated patients (Data are reported in percentages).

on the quality of life. In terms of health economics, a reduction in hospitalization associated with a reduced risk of adverse events corresponds to a reduction in direct and indirect health costs.

\section{Discussion}

Over 18 months, 438 consecutive patients were evaluated at the INTER-BOARD, with a progressive increase in the number of performed procedures. The INTERBOARD and a dedicated pathway for oncological patients optimized oncological procedures, especially in the field of interventional medicine; thus, progressively more specialists requested patient assessment and treatment at the IO center.

During INTER-BOARD meetings, specialists discussed the advantages and disadvantages of several potential procedures. These consensus procedures resulted in personalized treatment with tailored solutions. More specifically, in our cohort an impressive number of elderly patients was assessed and treated as a result of personalized management, aiming to avoid underand overtreatment. Among the optimized oncological procedures is for example radiotherapy, which offers IRT (brachytherapy; IRT) or electro-chemotherapy as 
a local treatment.[7] IRT represents the optimal method to apply a high radiation dose according to biological needs within the target volume including a rapid dose fall-off in adjacent organs at risk. Furthermore, relatively short treatment times and good functional outcomes are typical.[8]

Electrochemotherapy is a relatively new local tumor therapy. An electric field is generated in a tumor region between a set of electrodes. This electric field makes the cell membranes permeable to large molecules for a short time, thereby allowing anticancer drugs to enter cells.

At the same time, different focal treatments for example percutaneous or intra-arterial locoregional procedures are performed by interventional radiologists. Percutaneous locoregional treatments are mainly represented by radiofrequency ablation or microwave ablation, and cryoablation, which uses thermal energy to heat (radiofrequency, microwaves) or focally cool (cryoablation) tissues to cytotoxic levels (more than $60^{\circ} \mathrm{C}$ or $<-40^{\circ} \mathrm{C}$ ). Ablation technology has evolved rapidly during the past decades, with substantial technical and procedural improvements, which helped to improve clinical outcomes and safety profiles by creating larger areas of ablation, attempting to more precisely control the area of ablation, ensuring the safety of the procedure, and achieving greater long-term success.

Intra-arterial procedures are mainly represented by trans-arterial chemoembolization or embolization trans-arterial radio-embolization, in which devascularization/ischemia of tumors is associated with a selective and locoregional delivery of high chemotherapy drugs or internal radiotherapy (with radioactive isotopes e.g. yttrium-90 (Y90)-tagged glass or resin microspheres) dose to the cancer.[9-11]

Both, IRT and interventional radiology can be curative, converting patients from palliative to treatable status, or purely palliative. Furthermore, ancillary procedures, such as biopsy, fiducial marker placement, venous/vascular access, drainage, fluid management, and other procedures, are essential in cancer care, which are usually performed to permit other procedures or to treat complications. Regarding the role of specialists, the endoscopist can offer diagnostic procedures or ablative treatments using an endoscopic approach.

The role of geriatric oncologists is mandatory to evaluate patients' physical and cognitive performance, to choose the best therapeutic approach, and to avoid under- and overtreatment. Moreover, a geriatrician facilitates the identification of frail patients according to international guidelines, which is especially in times fearing coronavirus infection important.[12-14]
The most frequently treated cancer, accounting for $54 \%$ of all cancers treated, were endometrial and cervical cancers. This can be explained by the fact that our center is a hub of excellence for gynecological cancers. Thus, the most frequent procedures were IRT (47.9\%). Furthermore, the INTER-BOARD assessed also non-cancer patients (patients with keloid) and perform procedures that responded to non-cancer disease (treatment late toxicity), such as actinic proctitis (Endoscopic Argon Plasma Coagulation). Many researchers have tried to establish a relationship between waiting times and outcomes in the management of cancer patients. Most of these studies use waiting time until treatment initiation as a quality indicator. Using waiting time as a reference indicator allows us to improve access to treatments and to optimize treatments themselves.

Several studies suggested that discussed cases at an MDT were more likely to receive appropriate staging[15-18] and neo-adjuvant/adjuvant treatment compared to cases, which were not evaluated at an MDT. [19-22] A justification for this condition is represented by the hypothesis that the presence of more specialists, discussing a clinical case, allows a better and more precise cancer staging.[23] Moreover, the formulation of the best treatment plan is facilitated by the presence of several specialists discussing the advantages and disadvantages of each procedure applied to the patient in question.[24] Thus, multidisciplinary approaches are the best way to deliver complex cancer care to patients.[25] Both diagnostic capabilities and therapeutic options can be easily discussed to ensure the best treatment for each patient. All this can also lead to a non-standard treatment plan that adheres to existing guidelines and fulfills the needs of the individual patient. MDTs are thought to optimize patient outcomes and improve care performance, but it is a challenge that requires organizational and behavior changes. It is advisory that competent health managers who can improve effective teamwork within their organizations, lead these changes.

This approach offers the potential to achieve prolonged survival in patients who, in the past, would have only received palliative measures. For this reason, clinical guidelines have already begun to incorporate focal treatment techniques.[26] Oligometastatic status refers to a clinical stage in which patients present a limited number of metastases in a single organ or in few organs. These lesions may be more indolent than those typically observed in patients with multiple metastases. Oligometastatic patients have a better progno- 
sis with adequate treatment. Surgery has traditionally been the main treatment option, but, in many cases, these metastatic lesions are unresectable, or the patient is considered inoperable, or the long postoperative recovery period may require an unacceptable delay in the initiation of systemic therapy. As a result, less invasive treatments such as radiotherapy are often necessary. Technological advances of the past decades permitted high focal ablative doses with great precision to various sites. An important advantage of these high-dose treatments is that they require fewer fractions; thus, the treatment duration is shorter than with conventional fractionation or usual hypofractionation schedules.

INTER-BOARD meetings play a multifactorial role in bridging the gap between expert consensus panels and selection of the most appropriate care and optimize patient care by identifying all available options. For this reason, IO centers could have an important role for out-patient and in patient services, in education, as well as in clinical research.[27-31]

Stereotactic radiosurgery and stereotactic body radiation therapy are considered safe, with minimal treatment-related toxicity, only requiring a brief interruption of systemic therapy. The results of several heterogeneous randomized studies with predominantly small cohorts, as well as the analysis of prospective and retrospective studies, indicate that local ablative therapy in oligometastatic patients can improve the progression-free survival and the overall survival in a variety of tumor entities compared to systemic treatment alone. The best available evidence is on nonsmall-cell lung cancer and colorectal cancer.[32] Indeed, in recent years, it was demonstrated that local treatments (either radiotherapy or surgery) improve the progression-free survival and the overall survival in patients with oligometastatic non-small cell lung cancer at diagnosis and in those who respond to the initial systemic therapy. However, the association of locoregional interventional treatments and the abscopal effect was only addressed in few studies. The concept of abscopal effect is currently gaining importance in modern oncology; as the link between focal irradiation and triggering of immuno-mediated systemic antitumor effects is getting clearer.[33]

Although the results obtained are encouraging and suggest that a dedicated pathway for oncological patients can improve and optimize the treatment procedure, this study has some limitations: it is a retrospective analysis and due to the features with which the analysis and evaluations were conducted, efficacy data are absent.
Another limitation is represented by the fact that our patient population doesn't include all patients treated in our Institution with interventional locoregional treatments. In detail, INTER-BOARD is focalized on a selective subgroup of patients with a potential indication for locoregional treatments; however, it is not exclusive as other patients, not included in our evaluation, daily receive interventional locoregional treatments following indications from different multidisciplinary organ-specific tumor boards, well-active in our Institution. However, this limitation didn't affect our aim, to focused to register all patients treated in our institution but how a MTB could improve patient management.

\section{Conclusion}

IO represents one of the fastest-growing areas of interventional medicine and has become an essential part of comprehensive cancer care. Specialized MDT, such as the INTER-BOARD, play a multifactorial role to bridge the gap between expert consensus panels and appropriate care choice. MDTs can optimize patient care through identification of all available options, offer cross-specialty knowledge translation, provide the best evidence and continual updates on innovation among all stakeholders, ensure a low complication rate, and reduce waiting/hospitalization times. The results of our work shows how an IO center is fundamental to offer the best oncological treatments and to carry out a real personalization of treatments.

Peer-review: Externally peer-reviewed.

Conflict of Interest: All authors declared no conflict of interest.

Ethics Committee Approval: Ethics Committee Approval was waived due to the retrospective nature of the study and the anonymized data reported.

Financial Support: This study has received no financial support.

Authorship contributions: Concept - R.I., L.T.; Design - V.L., F.C.; Supervision - V.V., A.R., G.K., R.M., M.A.G.; Funding - None; Materials - None; Data collection and/or processing - F.A., A.L.; Data analysis and/or interpretation F.A., A.L.; Literature search - M.I., P.C.; Writing - M.I., B.F., P.C., A.C., G.C.; Critical review - R.I., B.F., P.C., M.I., V.L., A.C., F.A., A.L., F.C., F.M., G.C., A.R., G.K., M.A.G., R.M., V.V., L.T. 


\section{References}

1. Tagliaferri L, Vavassori A, Lancellotta V, De Sanctis V, Barbera F, Fusco V, et al. Can brachytherapy be properly considered in the clinical practice? Trilogy project: The vision of the AIRO (Italian Association of Radiotherapy and Clinical Oncology) Interventional Radiotherapy study group. J Contemp Brachytherapy 2020;12(1):84-9.

2. Yanazume Y, Yanazume S, Iio K, Yonekura R, Kojima N, Uchida N, et al. Major causes of impractical brachytherapy in elderly patients with uterine cervical cancer. J Obstet Gynaecol Res 2014;40(6):1725-32.

3. Colloca G, Tagliaferri L, Capua BD, Gambacorta MA, Lanzotti V, Bellieni A, et al. Management of the elderly cancer patients complexity: the radiation oncology potential. Aging Dis 2020;11(3):649-57.

4. Kovacs G, Tagliaferri L, Lancellotta V, Kovacs A, Iezzi $\mathrm{R}$, Gambarcorta MA, et al. Interventional oncology: should interventional radiotherapy (brachytherapy) be integrated into modern treatment procedures? Turk J Oncol 2019;34(Supp 1):16-22.

5. Kovács G, Tagliaferri L, Valentini V. Is an Interventional Oncology Center an advantage in the service of cancer patients or in the education? The Gemelli Hospital and INTERACTS experience. J Contemp Brachytherapy 2017;9(6):497-8.

6. Bretschneider T, Ricke J, Gebauer B, Streitparth F. Image-guided high-dose-rate brachytherapy of malignancies in various inner organs - technique, indications, and perspectives. J Contemp Brachytherapy 2016;8(3):251-61.

7. Crocetti L, Iezzi R, Goldberg SN, Bilbao JI, Sami A, Akhan $\mathrm{O}$, et al. The ten commandments of liver ablation: expert discussion and report from Mediterranean Interventional Oncology (MIOLive) congress 2017. Eur Rev Med Pharmacol Sci 2018;22(12):3896-904.

8. Malagari K, Iezzi R, Goldberg SN, Bilbao JI, Sami A, Akhan O, et al. The ten commandments of chemoembolization: expert discussion and report from Mediterranean Interventional Oncology (MIOLive) congress 2017. Eur Rev Med Pharmacol Sci 2018;22(2):372-81.

9. Bilbao JL, Iezzi R, Goldberg SN, Sami A, Akhan $\mathrm{O}$, Giuliante F, et al. The ten commandments of hepatic radioembolization: expert discussion and report from Mediterranean Interventional Oncology (MIOLive) congress 2017. Eur Rev Med Pharmacol Sci 2017;21(18):4014-21.

10. Wildes TM, O'Donovan A, Colloca GF, Cheung KL. Tumour boards in geriatric oncology. Age Ageing 2018;47(2):168-70.

11. Colloca G, Corsonello A, Marzetti E, Balducci L, Landi F, Extermann M, et al. Treating cancer in older and oldest old patients. Curr Pharm Des 2015;21(13):1699-
705.

12. Wildiers H, Heeren P, Puts M, Topinkova E, JanssenHeijnen ML, Extermann M, et al. International Society of Geriatric Oncology consensus on geriatric assessment in older patients with cancer. J Clin Oncol 2014;32(24):2595-603.

13. Freeman RK, Van Woerkom JM, Vyverberg A, Ascioti AJ. The effect of a multidisciplinary thoracic malignancy conference on the treatment of patients with lung cancer. Eur J Cardiothorac Surg 2010;38(1):1-5.

14. Davies AR, Deans DA, Penman I, Plevris JN, Fletcher J, Wall L, et al. The multidisciplinary team meeting improves staging accuracy and treatment selection for gastro-esophageal cancer. Dis Esophagus 2006;19(6):496-503.

15. Brännström F, Bjerregaard JK, Winbladh A, Nilbert M, Revhaug A, Wagenius G, et al. Multidisciplinary team conferences promote treatment according to guidelines in rectal cancer. Acta Oncol 2015;54(4):447-53.

16. Wille-Jørgensen P, Sparre P, Glenthøj A, Holck S, Nørgaard Petersen L, Harling $\mathrm{H}$, et al. Result of the implementation of multidisciplinary teams in rectal cancer. Colorectal Dis 2013;15(4):410-3.

17. Keating NL, Landrum MB, Lamont EB, Bozeman SR, Shulman LN, McNeil BJ. Tumor boards and the quality of cancer care. J Natl Cancer Inst 2013;105(2):113-21.

18. Boxer MM, Vinod SK, Shafiq J, Duggan KJ. Do multidisciplinary team meetings make a difference in the management of lung cancer? Cancer 2011;117(22):5112-20.

19. Palmer G, Martling A, Cedermark B, Holm T. Preoperative tumor staging with multidisciplinary team assessment improves the outcome in locally advanced primary rectal cancer. Colorectal Dis 2011;13(12):1361-9.

20. Santoso JT, Schwertner B, Coleman RL, Hannigan EV. Tumor board in gynecologic oncology. Int J Gynecol Cancer 2004;14(2):206-9

21. Wheless SA, McKinney KA, Zanation AM. A prospective study of the clinical impact of a multidisciplinary head and neck tumor board. Otolaryngol Head Neck Surg 2010;143(5):650-4.

22. Specchia ML, Frisicale EM, Carini E, Di Pilla A, Cappa $\mathrm{D}$, Barbara A, et al. The impact of tumor board on cancer care: evidence from an umbrella review. BMC Health Serv Res 2020;20(1):73.

23. Planchard D, Popat S, Kerr K, Novello S, Smit EF, Faivre-Finn C, et al. Correction to: Metastatic non-small cell lung cancer: ESMO Clinical Practice Guidelines for diagnosis, treatment and follow-up. Ann Oncol 2019;30(5):863-70.

24. Haussmann J, Matuschek C, Bölke E, Orth K, Ghadjar P, Budach W. The role of local treatment in oligometastatic and oligoprogressive cancer. Dtsch 
Arztebl Int 2019;116(50):849-56.

25. Tagliaferri L, Manfrida S, Barbaro B, Colangione MM, Masiello V, Mattiucci GC, et al. MITHRA - multiparametric MR/CT image adapted brachytherapy (MR/ CT-IABT) in anal canal cancer: a feasibility study. J Contemp Brachytherapy 2015;7(5):336-45.

26. Frakulli R, Galuppi A, Cammelli S, Macchia G, Cima $S$, Gambacorta MA, et al Brachytherapy in non melanoma skin cancer of eyelid: a systematic review. J Contemp Brachytherapy 2015;7(6):497-502.

27. Tagliaferri L, Bussu F, Fionda B, Catucci F, Rigante M, Gambacorta MA, et al. Perioperative HDR brachytherapy for reirradiation in head and neck recurrences: single-institution experience and systematic review. Tumori 2017;103(6):516-24.

28. Tagliaferri L, Garganese G, D'Aviero A, Lancellotta V, Fragomeni SM, Fionda B, et al. Multidisciplinary personalized approach in the management of vulvar cancer - the Vul.Can Team experience. Int J Gynecol Cancer 2020;30(7):932-8.

29. Tagliaferri L, Bussu F, Rigante M, Gambacorta MA, Autorino R, Mattiucci GC, et al. Endoscopy-guided brachytherapy for sinonasal and nasopharyngeal recurrences. Brachytherapy 2015;14(3):419-25.

30. Couñago F, Luna J, Guerrero LL, Vaquero B, GuillénSacoto MC, González-Merino T, et al. Management of oligometastatic non-small cell lung cancer patients: Current controversies and future directions. World J Clin Oncol 2019;10(10):318-39.

31. Fionda B, Massaccesi M, Tagliaferri L, Dinapoli N, Iezzi R, Boldrini L. Abscopal effect and interventional oncology: state of art and future perspectives. Eur Rev Med Pharmacol Sci 2020;24(2):773-6.

32. Valentini V, Maurizi F, Tagliaferri L, Cellini F. Spider: managing clinical data of cancer patients treated through a multidisciplinary approach by a palm based system. JPH 2008;5:66-76.

33. Tagliaferri L, Budrukkar A, Lenkowicz J, Cambeiro M, Bussu F, Guinot JL, et al. ENT COBRA ONTOLOGY: the covariates classification system proposed by the Head \& Neck and Skin GEC-ESTRO Working Group for interdisciplinary standardized data collection in head and neck patient cohorts treated with interventional radiotherapy (brachytherapy). J Contemp Brachytherapy 2018;10(3):260-6. 